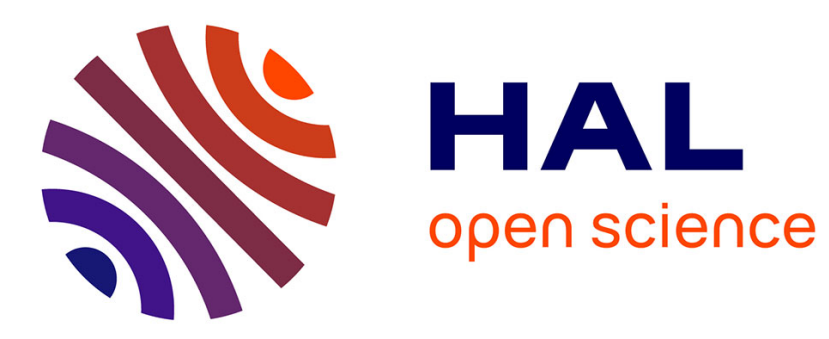

\title{
Tarif de cubage "peuplement" pour les mélèzes en Belgique méridionale.
}

Dominique Pauwels, Jacques Rondeux

\section{To cite this version:}

Dominique Pauwels, Jacques Rondeux. Tarif de cubage "peuplement" pour les mélèzes en Belgique méridionale.. Revue forestière française, 1999, 51 (5), pp.605-611. 10.4267/2042/5469 . hal03443360

\section{HAL Id: hal-03443360 \\ https://hal.science/hal-03443360}

Submitted on 23 Nov 2021

HAL is a multi-disciplinary open access archive for the deposit and dissemination of scientific research documents, whether they are published or not. The documents may come from teaching and research institutions in France or abroad, or from public or private research centers.
L'archive ouverte pluridisciplinaire HAL, est destinée au dépôt et à la diffusion de documents scientifiques de niveau recherche, publiés ou non, émanant des établissements d'enseignement et de recherche français ou étrangers, des laboratoires publics ou privés. 


\title{
technique et forêt
}

\section{TARIF DE CUBAGE “PEUPLEMENT" POUR LES MÉLĖZES EN BELGIQUE MÉRIDIONALE *}

\author{
Dominique PAUWELS - J. RONDEUX
}

Les tarifs de cubage "peuplement" permettent d'estimer directement le volume sur pied, ramené à l'hectare, d'un peuplement à partir de caractéristiques dendrométriques aisément récoltables telles que la hauteur dominante et la surface terrière. Ils s'avèrent particulièrement utiles dans les problèmes d'aménagement forestier et d'évaluation des ressources forestières, plus précisément lors d'estimations globales et rapides du matériel ligneux sur pied (Rondeux, 1977).

Plusieurs tarifs de ce type existent actuellement en Région wallonne pour les peuplements d'Épicéa (Rondeux, 1977), de Hêtre (Toussaint et al., 1983), de Douglas (Rondeux et al., 1991) et de Chênes indigènes cultivés en taillis-sous-futaie (Rondeux et al., 1987). À la suite d'une étude portant sur l'élaboration d'un modèle de croissance pour les mélèzes, nous avons mis à profit les nombreuses mesures dendrométriques récoltées au sein de placettes de production pour construire un tarif semblable adapté au cubage de peuplements de Mélèze, plus particulièrement de Mélèze du Japon [Larix kaempferi (Lamb.) Carr.] et ainsi étoffer la gamme de tarifs "peuplement" déjà existante.

Après un bref rappel de l'importance du Mélèze en Région wallonne (ci-dessous), nous présenterons le matériel d'étude et la méthode de construction du tarif (p. 606). Nous discuterons ensuite les résultats obtenus (p. 609), évoquerons la manière d'utiliser le tarif ( $p .609)$ et tirerons quelques conclusions (p. 610).

\section{LE MÉLÈZE EN RÉGION WALLONNE}

Au milieu des années 80 , selon l'inventaire forestier régional, la surface totale estimée des mélèzins [tous mélèzes confondus : Mélèze d'Europe (Larix decidua Mill.), Mélèze du Japon [Larix kaempferi (Lamb.) Carr.] et Mélèzes hybrides (Larix eurolepis)] avoisinait 10300 ha, soit approximativement

(*) Recherche financée par la Région wallonne, Ministère de l'Environnement, des Ressources naturelles et de l'Agriculture. 
$4 \%$ des peuplements résineux (Rondeux et al., 1986). Une mise à jour partielle de cet inventaire effectuée en 1996 (Lecomte et al., 1997) tend à montrer que les surfaces des mélèzins, comme celles des autres résineux, sont en légère régression.

La répartition des surfaces par classes d'âge en 1986 est présentée dans la figure 1 (ci-dessous). Plus de $75 \%$ des peuplements ont un âge compris entre 20 et 40 ans. Ces peuplements correspondent à la vague de plantation de Mélèze observée de 1950 à 1970.

Figure 1

RÉPARTITION PAR CLASSES D’ÂGE DES SURFACES DE MÉLÈZE EN RÉGION WALLONNE EN 1986

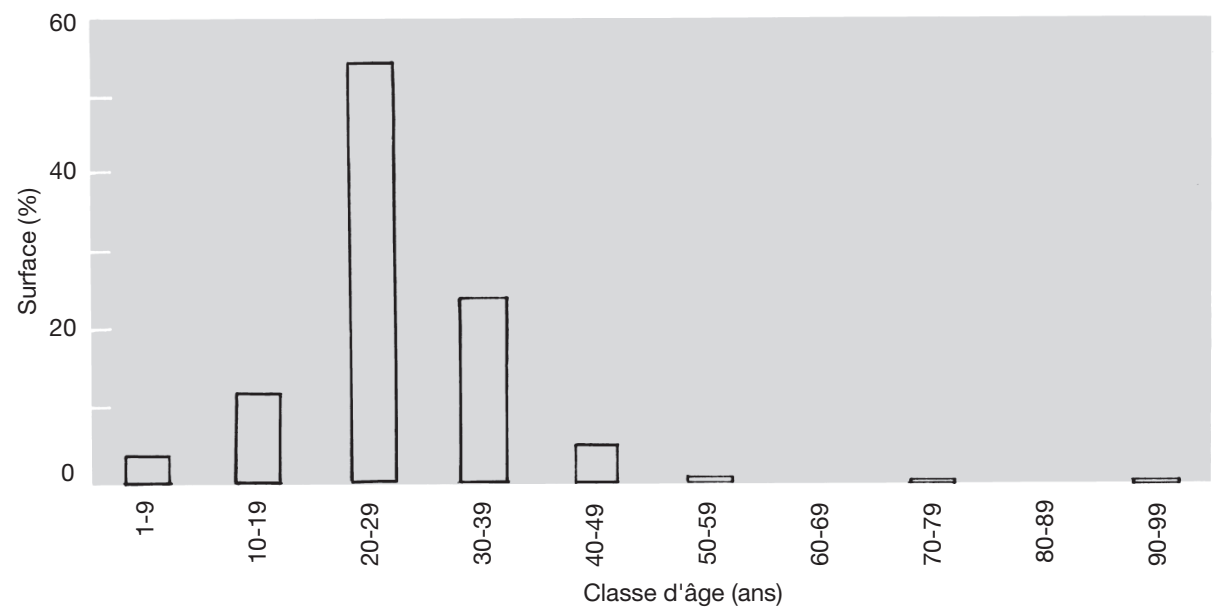

Les peuplements sont principalement situés sur des sols caillouteux à charge schisteuse, gréseuse ou schisto-gréseuse ( $80 \%$ ) et sont observés dans une très large gamme d'altitudes allant de 50 à $600 \mathrm{~m}$. L'Ardenne est la région naturelle qui comporte la majorité des mélèzins (50\% des peuplements observés) suivie par le Condroz $(29,1 \%)$ tandis que les autres régions sont moins concernées : Région limoneuse (7,3\%), Fagne-Famenne (9,2 \%), Région jurassique (4,4 \%).

II s'agit principalement de peuplements de Mélèze du Japon (environ $70 \%$ ). Les peuplements de Mélèze d'Europe et de Mélèzes hybrides (ces derniers étant encore très jeunes) sont également représentés mais dans une moindre proportion. Les peuplements ont une taille moyenne souvent réduite à quelques dizaines d'ares et sont très disséminés sur l'étendue de la Région wallonne.

\section{MATÉRIEL ET MÉTHODE}

Des placettes temporaires à surface variant de 1 à 22 ares (selon la densité et la surface des peuplements retenus dans l'échantillon) et représentant la plus grande diversité possible de conditions de sol et de croissance ont été installées dans 150 peuplements purs et équiennes de Mélèze situés en Région wallonne. Le choix de la localisation de ces placettes au sein des peuplements, d'étendues généralement faibles, a été largement dicté par la volonté d'éviter les effets de bordures et de trouées. II s'agit majoritairement (72 \%) de peuplements de Mélèze du Japon, le reste étant constitué de Mélèze d'Europe. La circonférence à $1,30 \mathrm{~m}\left(\mathrm{c}_{130}\right)$ de tous les arbres ainsi que la hauteur dominante (Hdom) du peuplement (correspondant par convention à la moyenne arithmétique des hauteurs des 100 plus gros bois à l'hectare ou des n plus gros bois par $\mathrm{n}$ ares de placette) 
ont été mesurées sur chaque placette. Seuls les peuplements dont la circonférence moyenne était supérieure à $50 \mathrm{~cm}$ ont été pris en considération, et ce afin que la majorité des arbres du peuplement puissent être cubés avec une précision suffisante.

À partir des variables précitées, le volume bois fort (volume géométrique de la tige depuis le sol jusqu'à la découpe de circonférence $22 \mathrm{~cm}: v 22$, en $\mathrm{m}^{3}$ ) de chaque arbre ayant une circonférence

Tableau I

Caractéristiques dendrométriques des peuplements de Mélèze mesurés en vue de la construction du tarif de cubage "peuplement"

\begin{tabular}{|l|c|c|c|c|}
\hline \multicolumn{1}{|c|}{ Caractéristiques dendrométriques } & Moyenne & Écart-type & Minimum & Maximum \\
\hline Circonférence moyenne $(\mathrm{cm}) \ldots \ldots \ldots \ldots$ & 87 & 21 & 51 & 141 \\
\hline Surface terrière $\left(\mathrm{m}^{2} / \mathrm{ha}\right) \ldots \ldots \ldots \ldots \ldots$ & 29,5 & 7,7 & 16 & 59 \\
\hline Hauteur dominante $(\mathrm{m}) \ldots \ldots \ldots \ldots \ldots$ & 24,5 & 4,3 & 14,3 & 33,8 \\
\hline Nombre de tiges à l'hectare $\ldots \ldots \ldots \ldots$ & 588 & 368 & 166 & 1650 \\
\hline Volume à l'hectare $\left(\mathrm{m}^{3} / \mathrm{ha}\right) \ldots \ldots \ldots \ldots$ & 320 & 97 & 163 & 655 \\
\hline
\end{tabular}

Tableau II Nombre de placettes ayant servi à construire le tarif de cubage "peuplement" réparties en catégories de surface terrière (G/ha en $\mathrm{m}^{2} / \mathrm{ha}$ ) et de hauteur dominante (Hdom en $\mathrm{m}$ )

\begin{tabular}{|c|c|c|c|c|c|c|c|c|c|c|c|}
\hline \multirow{2}{*}{$\begin{array}{c}\mathrm{G} / \mathrm{ha} \\
\left(\mathrm{m}^{2} / \mathrm{ha}\right)\end{array}$} & \multicolumn{11}{|c|}{ Hdom (m) } \\
\hline & $14-15$ & $16-17$ & $18-19$ & $20-21$ & $22-23$ & $24-25$ & $26-27$ & $28-29$ & $30-31$ & $32-33$ & Total \\
\hline $14-15$ & 0 & 0 & 0 & 0 & 1 & 0 & 0 & 0 & 0 & 0 & 1 \\
\hline $16-17$ & 0 & 0 & 0 & 1 & 0 & 0 & 1 & 0 & 0 & 0 & 2 \\
\hline $18-19$ & 0 & 0 & 1 & 0 & 1 & 0 & 1 & 2 & 0 & 0 & 5 \\
\hline $20-21$ & 0 & 0 & 1 & 0 & 1 & 6 & 2 & 3 & 1 & 0 & 14 \\
\hline $22-23$ & 0 & 0 & 2 & 2 & 2 & 4 & 3 & 2 & 0 & 0 & 15 \\
\hline $24-25$ & 2 & 0 & 0 & 1 & 3 & 6 & 1 & 3 & 1 & 1 & 18 \\
\hline $26-27$ & 2 & 2 & 1 & 3 & 2 & 3 & 4 & 0 & 0 & 0 & 17 \\
\hline $28-29$ & 3 & 0 & 0 & 3 & 3 & 1 & 5 & 2 & 2 & 3 & 22 \\
\hline $30-31$ & 0 & 0 & 0 & 0 & 1 & 2 & 1 & 1 & 0 & 0 & 5 \\
\hline $32-33$ & 0 & 1 & 0 & 4 & 1 & 2 & 3 & 3 & 1 & 0 & 15 \\
\hline $34-35$ & 0 & 1 & 2 & 1 & 1 & 2 & 0 & 1 & 0 & 0 & 8 \\
\hline $36-37$ & 0 & 0 & 1 & 4 & 1 & 0 & 2 & 1 & 1 & 0 & 10 \\
\hline $38-39$ & 0 & 0 & 2 & 1 & 1 & 0 & 0 & 0 & 1 & 0 & 5 \\
\hline $40-41$ & 0 & 0 & 0 & 1 & 0 & 0 & 0 & 0 & 0 & 0 & 1 \\
\hline $42-43$ & 0 & 0 & 0 & 0 & 0 & 1 & 1 & 1 & 1 & 0 & 4 \\
\hline $44-60$ & 0 & 0 & 0 & 1 & 2 & 1 & 1 & 3 & 0 & 0 & 8 \\
\hline Total & 7 & 4 & 10 & 22 & 20 & 28 & 25 & 22 & 8 & 4 & 150 \\
\hline
\end{tabular}


supérieure à $22 \mathrm{~cm}$ a été calculé grâce à l'équation de cubage "arbre" de Dagnelie et al. (1999) dont le domaine de validité englobe la région étudiée. Cette équation qui fait intervenir la circonférence à 1,30 $\mathrm{m}\left(c_{130}\right.$, en $\left.\mathrm{cm}\right)$ et la hauteur dominante $(\mathrm{Hdom}$, en $\mathrm{m})$ a été établie à partir d'arbres abattus dont les volumes résultent d'un cubage par billons successifs d'une longueur d'un mètre dans les 10 premiers mètres de la tige et de 2 mètres au-delà. Elle se présente sous la forme suivante :

$$
\begin{gathered}
\mathrm{v} 22=-0,17511 * 10^{-3}+0,50499 * 10^{-3}{ }^{*} c_{130}+0,15638 * 10^{-4}{ }^{*} c_{130}{ }^{2} \\
-0,74426 * 10^{-7 *} c_{130}{ }^{3}-0,21894 * 10^{-2}{ }^{*} \text { Hdom }+0,32361 * 10^{-5 *} c_{130}{ }^{2} \text { Hdom }
\end{gathered}
$$

Le volume est alors ramené à l'hectare puis mis en relation avec la surface terrière et la hauteur dominante du peuplement. Étant donné l'existence d'un tarif de cubage "arbre" unique pour les deux espèces, le tarif de cubage "peuplement" concerne indistinctement les Mélèzes d'Europe et du Japon.

Les caractéristiques dendrométriques générales des placettes ayant servi à construire le tarif de cubage "peuplement" sont reprises, en termes de moyennes et de variabilité, dans le tableau I (p. 607). Le tableau II (p. 607) présente le nombre de placettes par catégories de surface terrière $(\mathrm{G} / \mathrm{ha}$ ) et de hauteur dominante (Hdom). II fournit ainsi de précieuses indications sur le domaine de validité du tarif.

Tableau III Volume ( $\mathrm{m}^{3} / \mathrm{ha}$ ) du bois fort de la tige (jusqu'à la découpe de $22 \mathrm{~cm}$ de circonférence) en fonction de la surface terrière (G/ha en $\mathrm{m}^{2} / \mathrm{ha}$ ) et de la hauteur dominante (Hdom en $\mathrm{m}$ ) Les valeurs grisées correspondent à des classes de surface terrière et de hauteur dominante non représentées dans l'échantillon

\begin{tabular}{|c|c|c|c|c|c|c|c|c|c|c|}
\hline \multirow{2}{*}{$\begin{array}{c}\text { G/ha } \\
\left(\mathrm{m}^{2} / \mathrm{ha}\right)\end{array}$} & \multicolumn{10}{|c|}{ Hdom $(\mathrm{m})$} \\
\cline { 2 - 12 } & 15 & 17 & 19 & 21 & 23 & 25 & 27 & 29 & 31 & 33 \\
\hline 15 & 113 & 125 & 136 & 147 & 159 & 170 & 181 & 193 & 204 & 216 \\
\hline 17 & 127 & 140 & 153 & 166 & 179 & 192 & 204 & 217 & 230 & 243 \\
\hline 19 & 141 & 155 & 170 & 184 & 199 & 213 & 227 & 242 & 256 & 271 \\
\hline 21 & 155 & 171 & 187 & 203 & 219 & 235 & 250 & 266 & 282 & 298 \\
\hline 23 & 169 & 186 & 204 & 221 & 239 & 256 & 273 & 291 & 308 & 326 \\
\hline 25 & 183 & 201 & 220 & 239 & 258 & 277 & 296 & 315 & 334 & 353 \\
\hline 27 & 196 & 217 & 237 & 258 & 278 & 299 & 319 & 340 & 360 & 381 \\
\hline 29 & 210 & 232 & 254 & 276 & 298 & 320 & 342 & 364 & 387 & 409 \\
\hline 31 & 224 & 248 & 271 & 295 & 318 & 342 & 365 & 389 & 413 & 436 \\
\hline 33 & 238 & 263 & 288 & 313 & 338 & 363 & 388 & 414 & 439 & 464 \\
\hline 35 & 252 & 278 & 305 & 332 & 358 & 385 & 411 & 438 & 465 & 491 \\
\hline 37 & 266 & 294 & 322 & 350 & 378 & 406 & 434 & 463 & 491 & 519 \\
\hline 39 & 280 & 309 & 339 & 369 & 398 & 428 & 457 & 487 & 517 & 546 \\
\hline 41 & 294 & 325 & 356 & 387 & 418 & 449 & 480 & 512 & 543 & 574 \\
\hline 43 & 307 & 340 & 373 & 405 & 438 & 471 & 503 & 536 & 569 & 601 \\
\hline 45 & 321 & 355 & 390 & 424 & 458 & 492 & 526 & 561 & 595 & 629 \\
\hline
\end{tabular}




\section{RÉSULTATS OBTENUS}

La relation exprimant le volume (jusqu'à la découpe de circonférence $22 \mathrm{~cm}$ ) ramené à l'hectare (V22, en $\left.\mathrm{m}^{3} / \mathrm{ha}\right)$ en fonction de la surface terrière à l'hectare $\left(G\right.$, en $\mathrm{m}^{2} /$ ha) et la hauteur dominante (Hom, en $\mathrm{m}$ ) résulte d'un ajustement par la méthode des moindres carrés non pondérés. L'équation finale se présente sous la forme mathématique suivante :

$$
\text { V22 }=9,031+0,37987 * G * H d o m+1,240078 * G \quad[R=99,9 \%, C V R=30,3 \%]
$$

et donne lieu aux résultats fournis dans le tableau III (p. 608).

Les résidus de cette équation sont, dans $90 \%$ des cas, compris entre -4 et $4 \mathrm{~m}^{3} / \mathrm{ha}$ et présentent un minimum de $-11,5 \mathrm{~m}^{3} /$ ha et un maximum de $7,2 \mathrm{~m}^{3} /$ ha. On observe qu'ils sont toujours inférieurs en valeur absolue à $2,5 \%$ du volume observé. Rappelons que le calcul des volumes des peuplements résulte de l'application d'un tarif de cubage "arbre", ce qui a largement contribué à lisser la variabilité réelle.

À titre purement indicatif, à surface terrière et hauteur dominante égales, le volume sur pied (jusqu'à la découpe de circonférence $22 \mathrm{~cm}$ ) des peuplements de Mélèze est toujours supérieur à celui de l'Épicéa commun.

Afin de valider le tarif de cubage "peuplement", celui-ci a été testé sur un échantillon de 65 peuplements différents de ceux ayant servi à sa construction. Les volumes de ceux-ci ont été estimés à partir de placettes temporaires pour lesquelles chaque arbre a été cubé via le tarif de cubage "arbre" déjà mentionné. Ils ont été comparés aux estimations fournies par le tarif de cubage "peuplement". L'erreur d'estimation est, dans $90 \%$ des cas, inférieure à $2 \%$ en valeur absolue avec un maximum de $3,7 \%$.

Si on privilégie la rapidité d'estimation, ce qui revient à accepter une perte de précision, la formule classique de cubage de peuplement

$$
V=V 22=G *{ }^{*} \text { dom }{ }^{*} F
$$

dans laquelle $\mathrm{F}$ représente le coefficient de forme moyen du peuplement, pourrait également être utilisée. En effet, l'ajustement des valeurs V/G en fonction de la hauteur dominante montre que le coefficient de forme $\mathrm{F}$ se caractérise par une valeur moyenne très stable de 0,446 (1) (écart-type de 0,014 ) pour des hauteurs dominantes s'échelonnant de 14 à $34 \mathrm{~m}$. L'utilisation de cette valeur moyenne pour estimer très rapidement le volume des peuplements ayant servi à construire et à valider la table de cubage entraîne une erreur inférieure à $2 \%$ dans $50 \%$ des cas et inférieure à $5 \%$ dans $90 \%$ des cas.

\section{UTILISATION}

L'utilisation proprement dite du tarif en gestion forestière courante nécessite évidemment l'estimation préalable de la hauteur dominante et de la surface terrière du peuplement.

Si celui-ci n'est pas homogène du point de vue de ses propriétés stationnelles (sol), il sera nécessaire de le décomposer en strates (zones plus homogènes) et de procéder à des estimations différenciées de la hauteur dominante. Cette dernière sera mesurée, dans chaque strate, en plusieurs

(1) Afin d'éviter toute ambiguïté, le coefficient de forme $\mathrm{F}$ utilisé ici est basé sur la hauteur dominante, il n'est donc pas étonnant que, pour un même peuplement, il soit inférieur au coefficient de forme généralement calculé à partir de la hauteur moyenne $\mathrm{H}$. À titre d'information, pour l'Épicéa commun en Ardenne, le même type de raisonnement a conduit à un coefficient de forme $F$ de 0,421 (Rondeux, 1977). 
points (endroits choisis aléatoirement et en nombres liés à l'hétérogénéité du peuplement). On calculera la moyenne des hauteurs totales des arbres les plus gros, chacun d'entre eux étant repéré au sein d'une placette de 1 are (rayon approximatif de $6 \mathrm{~m}$ ). L'utilisation d'un dendromètre ultrasonique tel que le Forestor Vertex garantira une mesure à la fois rapide et précise, y compris dans des conditions de mesure éventuellement difficiles (densité, visibilité) (Rondeux et Pauwels, 1998).

Quant à la surface terrière, elle sera mesurée aux mêmes points que ceux auxquels la hauteur dominante se réfère. Pour des raisons de rapidité de mesure, on conseillera de l'estimer en utilisant un prisme relascopique à facteur de surface terrière adapté à la densité des peuplements (facteur 1 pour des surfaces terrières inférieures à $20 \mathrm{~m}^{2} /$ ha et facteur 2 pour des surfaces terrières supérieures à cette même valeur). On veillera à respecter les élémentaires précautions d'usage (Rondeux, $1983,1993)$ destinées à limiter l'effet "opérateur" sur la qualité des estimations. Celles-ci pourraient évidemment aussi résulter de mesures individuelles de grosseur au sein de placettes d'échantillonnage à surfaces définies, mais on pourrait alors se demander pourquoi ne pas préférer un tarif "arbre".

La mise en œuvre du tarif de cubage proposé n'est valable que pour des peuplements situés en Région wallonne et présentant des caractéristiques comparables à celles rassemblées dans le tableau I (p. 607).

\section{CONCLUSIONS}

Un tarif de cubage a été déterminé afin de calculer directement le volume sur pied (jusqu'à la découpe de circonférence $22 \mathrm{~cm}$ ) à l'hectare de peuplements de Mélèze à partir de leur surface terrière et de leur hauteur dominante. La précision de ce tarif est très satisfaisante puisque l'erreur est inférieure à $2 \%$ dans $90 \%$ des cas d'estimation. Le tarif peut être utilisé sans crainte pour des estimations rapides de volume lors de la réalisation d'inventaires de gestion ou d'évaluations financières. Le cubage rapide des peuplements peut également être réalisé, si on admet une légère perte de précision, en utilisant un coefficient de forme "peuplement" constant de 0,446.

II est par ailleurs recommandé de n'utiliser ces tarifs que pour des peuplements dont la circonférence moyenne dépasse $50 \mathrm{~cm}$.

\begin{tabular}{|c|}
\hline $\begin{array}{c}\text { Dominique PAUWELS } \\
\text { Assistante de Recherche }\end{array}$ \\
Jnité de Gestion et Économie forestières \\
FACULTÉ UNIVERSITAIRE \\
DES SCIENCES AGRONOMIQUES DE GEMBLOUX \\
Passage des Déportés, 2 \\
B-5030 GEMBLOUX (BELGIQUE) \\
\hline
\end{tabular}

\section{Remerciements}

II nous est agréable de remercier les personnes qui ont contribué à la réalisation de cette étude et plus particulièrement :

- MM. Delisee, Florkin, Havenne, Larose, Marenne, Morimont et Thirion, techniciens forestiers, qui ont sillonné la Région wallonne, repéré les peuplements de Mélèze et effectué toutes les mesures de terrain.

- les propriétaires forestiers privés ainsi que les agents de la Division Nature et Forêts qui nous ont permis, grâce à leur collaboration, de rassembler une quantité très importante d'informations utiles à une meilleure connaissance du Mélèze en Région wallonne. 


\section{Technique et forêt}

\section{BIBLIOGRAPHIE}

DAGNELIE (P.), PALM (R.), RONDEUX (J.), THILL (A.). - Tables de cubage des arbres et des peuplements forestiers. - Gembloux : Les Presses Agronomiques, 1999. - 128 p.

LECOMTE (H.), FLORKIN (P.), THIRION (M.). - L'Inventaire des massifs forestiers de la Wallonie : aperçu global de la situation en 1996. - Namur : Ministère de la Région wallonne - Direction générale des Ressources naturelles et de l'Environnement, 1997. - 43 p. (Fiche technique ; 9).

RONDEUX (J.). - Construction et utilisation de tarifs de cubage “peuplements" pour l’Épicéa en Ardenne Méridionale. - Bulletin des Recherches agronomiques de Gembloux, vol. 12, n 4, 1977, pp. 339-348.

RONDEUX (J.). - La Mesure des arbres et des peuplements forestiers. - Gembloux : Les Presses Agronomiques, 1993. - $521 \mathrm{p}$.

RONDEUX (J.). - La Méthode de l'angle critique : une conception particulière de l'échantillonnage appliquée aux inventaires forestiers. - Annales de Gembloux, vol. 89, $\mathrm{n}^{\circ} 3$, 1983, pp. 183-200.

RONDEUX (J.), HEBERT (J.), TOUSSAINT (A.). - L'Estimation rapide des volumes sur pied en taillis-sous-futaie de chênes. - Bulletin des Recherches agronomiques de Gembloux, vol. 22, n 3, 1987, pp. 199-207.

RONDEUX (J.), LAURENT (C.), LEJEUNE (P.). - Tarifs de cubage peuplement pour le Douglas (Pseudotsuga menziesii (Mirb.) Franco) en Moyenne et Haute Belgique. - Revue forestière française, vol. XLIII, $n^{\circ}$ 6, 1991, pp. 507-513.

RONDEUX (J.), LECOMTE (H.), FAGOT (J.), LAURENT (C.), TOUSSAINT (A.). - Quelques données statistiques récentes sur la forêt wallonne. - Bulletin de la Société royale forestière de Belgique, vol. 93, $n^{\circ} 1,1986$, pp. 1-22.

RONDEUX (J.), PAUWELS (D.). - Le Forestor Vertex : une nouvelle génération de dendromètres. - Revue forestière française, vol. L, $\mathrm{n}^{\circ} 1,1998$, pp. 59-64.

TOUSSAINT (A.), FAGOT (J.), LECOMTE (H.), RONDEUX (J.). - Tarif de cubage peuplement pour le Hêtre (Fagus sylvatica L.) en Wallonie. - Bulletin des Recherches agronomiques de Gembloux, vol. 18, n 3, 1983, pp. 165172.

\section{TARIF DE CUBAGE “PEUPLEMENT” POUR LES MÉLĖZES EN BELGIQUE MÉRIDIONALE (Résumé)}

Des données dendrométriques récoltées dans 150 placettes temporaires installées dans des peuplements équiennes de Mélèze situés en Belgique méridionale (Région wallonne) ont permis de construire un tarif de cubage "peuplement" exprimant le volume bois fort tige (jusqu'à la découpe de circonférence $22 \mathrm{~cm}$ ) à l'hectare de mélèzins en fonction des surfaces terrières et des hauteurs dominantes qui les caractérisent.

\section{"STAND" VOLUME TABLES FOR LARCHES IN SOUTHERN BELGIUM (Abstract)}

Dendrometric data taken from 150 temporary plots set up in even-aged stands of larch trees in southern Belgium (French-speaking region) were used to devise a "stand" volume table that expresses the stem solid volume (for log girths not less than $22 \mathrm{~cm}$ ) per hectare of larch depending on the land surface areas and dominant tree heights. 